\title{
Prosthetic Foot Design: The Significance of the Normalized Ground Reaction Force
}

A.Y. Bani Hashimı, N.A. Abu Osman2, W.A.B. Wan Abas2, and L. Abdul Latif3

1 Department of Robotics \& Automation, Faculty of Manufacturing Engineering, Universiti Teknikal Malaysia Melaka, Durian Tunggal, 76100 Melaka, Malaysia

2 Department of Biomedical Engineering, Faculty of Engineering, University of Malaya, Kuala Lumpur, Malaysia

3 Department of Rehabilitation Medicine, Faculty of Medicine, University of Malaya, Kuala Lumpur, Malaysia

\section{INTRODUCTION}

In Malaysia, there is a need to produce lower limb prosthetic

that is affordable. At present, good prosthetic feet are

mostly the import products. A typical foot prosthesis costs

around RM8,000. This is comparable to the price of a motorcycle.

In fact, a number of young male patients lost their

lower limb due to motorcycle accidents! Unluckily for

them, it is like losing a motorcycle and limb, and as a replacement

they need to purchase prosthetic foot.

This work tries to replicate biological foot system and develop a model to be used as reference in design and development of prosthetic foot. It begins by investigating kinematic structure of human foot - why the bones and joints are arranged that way, why they are different from other primates, and how can they be imitate to develop foot prosthesis. It poses a challenge to emulate biological systems, which seem to have the least flaws.

At present there are no studies that explain the functions of the point of contact (POC) - the point that experiences the vertical ground reaction forces. There are, however, studies on foot biomechanics such as in [1], and on walking gait analysis in [2]-[8], and [9]. Computational intelligence 
is the current trend in gait analysis [10]. The advantage of computational intelligence technology is that it offers the ability to investigate non-linear data relationships.

The scope of the design follows the notion of energy storage and retrieval capabilities. This is achievable by applying a flexible keel. The complete prosthetic foot design has the foot-ankle module attaches to the flexible keel. Thus, the objectives of this work are to identify bones, joints, and ground reaction forces localities that act on specific bones, to design the way to visualize them numerically, to develop methods in determining foot identity, and to assess the foot identity to build custom prosthetic foot.

\section{MetHod}

The first step is to study the anatomy of foot. Then, a graph is created that charts bones and joints localities. Vertex symbolizes bone, whereas as an edge symbolizes joint. The graph eventually characterizes foot. It depicts bones adjacencies, bones and joints incidences, and the routes of paths from the root. This is further elaborated in Fig. 1.

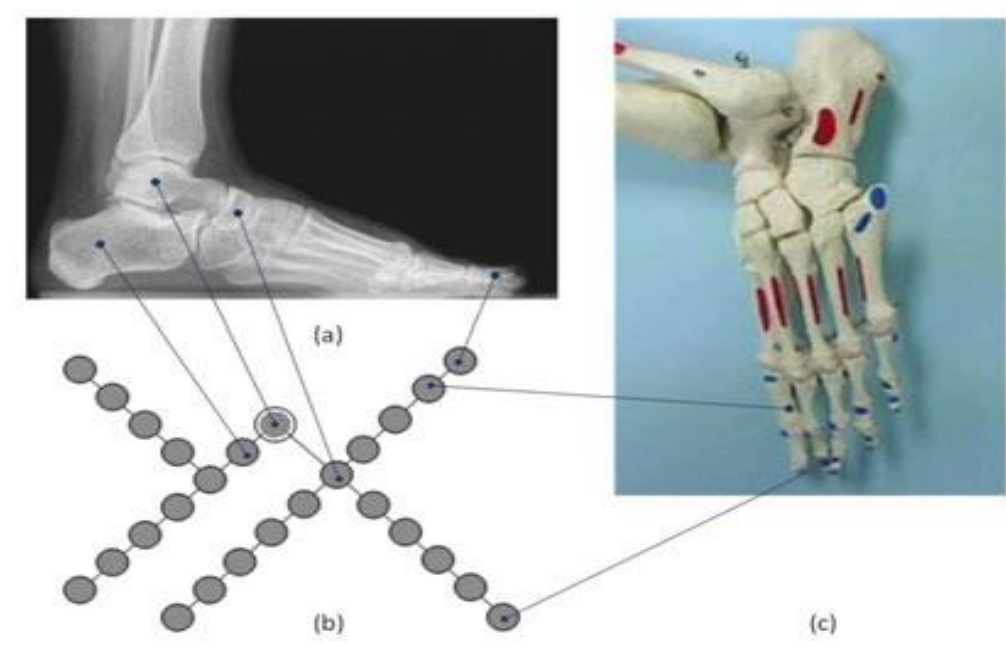

Fig. 1(a) The foot radiograph and we propose the graph as shown in Fig. 1(b). Figure 1(c) is the model skeleton. Six vertices pinpoint their assigned bones in Fig. 1(a) and Fig. 1(c) as examples. Graphs can aid the process of mechanical structures design [12] 


\section{A. Mathematical Model}

By inspection, human foot has twenty-seven bones. It

has five digits known as the phalanges. They control the drop-off- the experience at the end of gait. Calcaneus, on the other hand, works upon gait initiation. It is the largest bone that bears body weight upon heel strike. Navicular and cuboid bear distributed body weight upon foot flat. The last phalanx or the toe bears the body weight at the end of gait. In Fig. 1, the circles are the vertices that represent bones and the connecting lines are the edges that represent joints. The concentric circle is the root, which is the talus.

Let there be a number of sequences from POC-0 to POC-

13 during gait. The sequence $Q$ follows the POCs. Every sequence has at least one POC and a GRF- $k f$ except for ${ }_{0} Q$ and ${ }_{13} Q$. On foot flat, there are twelve POCs. This is shown in Fig. 2.

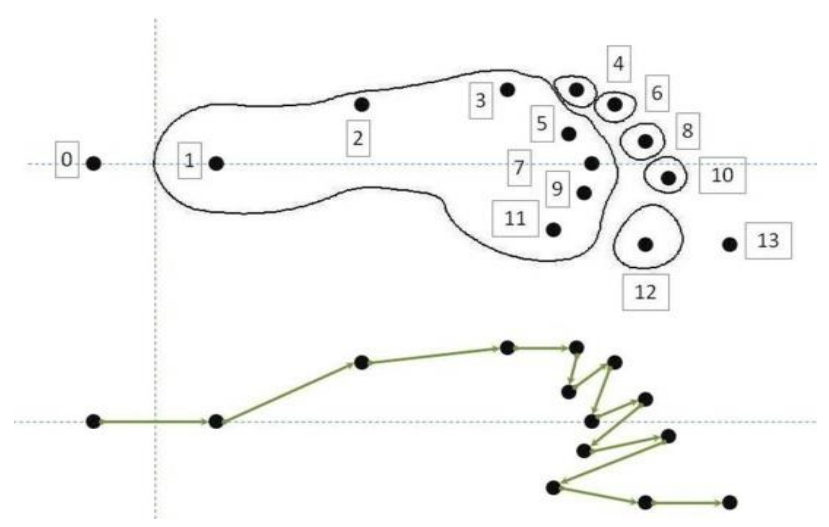

Fig. 2 Above - the point-of-contacts shown on the footprint. Below- the ambulatory path

It is straightforward that POC-0 in ${ }_{0} Q$ does not experience any reaction prior to touching the ground. In this sequence, none of the POC has contact with the ground. This is also true for the last sequence. However, upon heel strike POC-1 bears maximum body load. Equation (1) explains the process above where the rows are the sequences and the columns are the POC. The degree ' $\mathrm{V}$ ' means very 
large, 'L' large, 'M' medium, 'S' small, and 'A' absent. In ${ }_{0} Q$ there is no GRF and ${ }_{1} Q$ has very large GRF upon heel strike. For ideal gait, the computed ' $M$ '-curve is shown in Fig. 3, which is the basis for selection of the active POCs. These POCs lie within the curve's peaks and valley.

Full text is available at :

http://download.springer.com/static/pdf/535/chp\%253A10.1007\%252F978-3-642-21729-

6_187.pdf?auth66=1393724488_78d6437ca2e8f6455fa2b71566e1ba10\&ext $=. p d f$

http://link.springer.com/chapter/10.1007/978-3-642-21729-6_187 